\title{
VERZEICHNIS DER VON H. FRUHSTORFER \\ IN TONKIN GESAMMELTEN MELOLONTHIDEN
}

von J. Voser.

1. Serica nigroguttata BRsk., Berlin. ent. Zeitschr., 1897, p. 389. - Montes Mauson. - Bei den Exemplaren von Tonkin stehen die schwarzen Flecke auf den Flügeldecken viel weitlüufiger als bei den typischen Exemplaren von Kiukiang, so dass die Flügeldecken viel heller erscheinen und die beiden scharzen Makel an den Endbuckeln deutlich hervortreten. Die Schuppen der Oberseite sind kräftiger als bei den typischen Exemplaren und die an den Seiten der Brust sind weniger borstenartig.

2. Antoserica hongkongica Brsk., Berlin. ent. Zeitschr., 1898, p. 208. - Montes Mauson. - Die Exemplare von Tonkin sind durchschnittlich etwas grösser als die typischen von Hongkong, doch kann ich sonst keinen Unterschied finden.

3. Antoserica rubescens n. sp. - Rufobrunnea, opaca, opalescens. Clypeo nitidissimo, sparsim ruguloso-punctato, marginibus elevatis, angulis anticis rotundatis, medio longitudinaliter subcarinato; fronte laxe subtiliter punctata; antennis rufis, 10 -articulatis; prothorace haud dense subtiliter punctulato, punctis minutissime setosis; elytris striatis, interstitiis punctatis, punctis setas minutas ferentibus; pygidio triangulari, apice rotundato, lateribus sinuatis. Subtus medio sparsim, pectoris lateribus sat dense umbilicato-punctatis, abdomine seriatim transverse-setoso; tibiis posticis dilatatis et abbreviatis. - Long. 8-9 mill. - Montes Mauson.

Der Antoserica flammea BRsk. von Hongkong in Gestalt und Färbung sehr ähnlich, durch den viel schwächer runzelig punktierten und sehr glänzenden Clypeus, sowie durch die viel breiteren Hinterschenkel und Hinterschienen von dieser Art leicht zu unterscheiden.

Die Färbung ist hell rotbraun mit schwachem Opalglanz. Das Kopfschild ist breit, umrandet, vorn gerade abgestutzt, die Vorderecken sind flach gerundet, die Mitte ist schwach gekielt. Die Punktierung ist runzelig, aber flach und weitläufig, die Oberfläche ist sehr glänzend. Die Punktierung der Stirn ist wegen der Tomentbekleidung nur sehr undeutlich. Die hellbraunen Fühler sind zehngliederig, der Fächer ist so lang wie der Stiel. Das Halsschild ist doppelt so breit als lang, leicht gewölbt, die Seiten gleichmässig 
gerundet und beborstet. Die Oberfläche ist fein punktiert, die Punkte sind mit sehr kleinen Börstchen versehen. Das Schildchen ist dreieckig mit stumpfer Spitze. Die Flügeldecken sind in Reihen punktiert, von den unregelmässig punktierten Zwischenräumen sind die seitlichen schwach gewölbt. Die Punkte tragen winzige Börstchen, die Seitenränder der Flügeldecken sind abstehend beborstet. Das Pygidium ist dreieckig, mit abgerundeter Spitze und concaven Seitenrändern. Die Seiten der Brust sind ziemlich dicht mit Nabelpunkten bedeckt. Die Mitte der Brust zeigt einige längere Borsten und jedes Abdominalsegment trägt eine Querreihe von beborsteten Punkten. Die flachen Hinterschenkel und Hinter. schienen sind ziemlich stark verbreitert, letztere auch sehr verkürzt.

4. Antoserica birmanica Brsk., Berl. ent. Zeitschr., 1898, p. 343. - Montes Mauson, Chiem-Hoa. - Da mir diese Art auch aus Assam vorliegt, so glaube ich, dass sie mit der nur sehr unvollkommen beschriebenen bimaculata Hope identisch ist.

5. Gephaloserica parva n. sp. - Brunnea, nitida. Clypeo grosse punctato, marginibus elevatis, antice trilobato; fronte minus dense punctata; antennis flavis, 10-articulatis, flabello 3 -articulato; prothorace lato, antice rotundato-attenuato, sat dense punctato, lateribus flavo-ciliatis, scutello magno, triangulari; elytris irregulariter punctatis, subcostatis, lateribus setosis; pygidio convexo, rugoso-punctato, apice ciliato. Subtus medio sparsim, pectoris lateribus dense punctatis; tibiis anticis acute bidentatis, femoribus tibiisque posticis dilatatis, fere lævibus. - Long. 6 mill. - ChiemHou.

Wegen des dreizähnigen Vorderrandes des Clypeus habe ich die Art der Gattung Ciephaloserica zugeteilt : Diese wurde von Brenske erst nachträglich aufgestellt und müssen einige in der Monographie als Antoserica oder Serica beschriebene Arten auf diese Gattung übertragen werden. Die Art hat Aenlichkeit mit Autoserica setosa Brsk., von der sie sich jedoch sofort durch die Form des Clypeus unterscheidet.

Gelbbraun, schwach glänzend. Der Clypeus ist grob punktiert, nach vorn verschmälert, die Ränder sind aufgebogen, der Vorderrand ist stumpf dreispitzig. Die gelbbraunen Fühler sind zehngliederig, der dreigliederige Fächer ist fast so lang wie der Stiel. Das Halsschild ist ziemlich dicht und fein punktiert, nach vorn verjüngt, die Seiten sind gleichmässig gerundet und gelbbraun bewimpert. Das Schildchen ist gross, dreieckig mit stumpfer Spitze, mit Ausnahme eines kleinen Mittelfleckes punktiert. Die Flügeldecken 
sind unregelmässig punktiert, schwach gestreift, die weniger dicht punktierten Zwischenräume namentlich an den Seiten schwach gewölbt, die Seitenränder mit abstehenden Borstenhaaren besetzt. Das Pygidium ist runzelig, am Hinterrande bewimpert. Die Seiten der Brust sind grob punktiert, die Mitte derselben ist mit einigen Borstenhaaren besetzt und mit einer Längsfurche versehen. Die Vorderschienen sind kräftig zweizähnig, die Hinterschenkel und Hinterschienen sind nur wenig verbreitert und fast unpunktiert.

6. Neoserica ursina Brsk., Mémoires Soc. Ent. de Belgique, II, 1894, p. 49. - Montes Mauson. - Die Exemplare von Tonkin stimmen mit den typischen Exemplarer von Shanghai überein. Bei einem Exemplar, welches rotbraun gefärbt ist, fehlen sowohl die abstehenden Haare des Kopfes, als auch die abstehenden Borsten der Flügeldecken.

7. Neoserica vulpina n. sp. - N. ursinae Brsk. affinis. Castanea, opaca, rufobrunneo-pilosa et setosa. Capite piloso, vertice opaco, clypeo nitido, punctato, lato, margine antico paulo exciso, angulis anticis rotundatis; antennis ferrugineis, clava picea, maris 4-, feminea 3 - articulata; prothorace longitudine latiore, antice paulo attenuato, rufobrunneo-villoso; elytris striatis, interstitiis subconvexis, sparsim ferrugineo-pilosis et brunneo-setosis; pygidio corporeque infra hand dense fulvo-pilosis. - Long. : 11 mill. - Montes Mauson.

Mit Neoserica ursina BRSk. nahe verwandt. Grösser, die Färbung brauner, die abstehenden Haare der Oberseite und die Borsten nicht greis, sondern braun.

Der Clypeus ist glänzend, seine Vorderecken sind breit gerundet, der Vorderrand ist schwach ausgeschweift. Er ist mit nicht dicht stehende Nabelpunkten bedeckt, welche abstehende Borstenhaare tragen. Die wenig deutliche Naht ist bogenförmig. Die Fühler sind zehngliederig, der Fächer ist beim c vier- beim $q$ drei-gliederig. Das Halsschild ist am Hinterrande fast doppelt so breit als lang, vor dem Vorderrande verschmälert, die Vorderecken sind nicht vorgezogen. Es ist mit zerstreuten anliegenden gelblichen Haaren, sowie mit abstehenden langen gelbbraunen Haaren bedeckt. Das Schildchen ist etwas dichter anliegend behaart. Die Flügeldecken sind schmal gestreift, die Zwischenräume schwach gewölbt, zerstreut und anliegend gelbbraun behaart und mit kräftigen langen braunen Borsten, die reihenförmig in den Zwischenräumen stehen, versehen. Pygidium und Unterseite sind mässig dicht gelblich behaart. Auch bei dieser Art sind die Hinterschienen ebenso wie bei ursina schlanker, als es sonst bei den Arten der Gattung Neoserica der Fall ist. 
8. Neoserica nigrosetosa n. sp. - Nigra, opaca, cinereo- et nigro-pilosa, elytris nigro-setosis. Clypeo nitido, setoso-punctato, fronte opaca, cinereo-pilosa et nigro-setosa; antennis flavis, clava nigra, maris 4- flabellata; prothorace longitudine multo latiore, antice attenuato, haud dense cinereo-piloso et selis nigris erectis ornato; elytris striatis, interstitiis subconvexis, sparsim cinereopilosis et longe nigro-setosis; pygidio corporeque infra cinereopilosis. - Long. : 7 mill. - Montes Mauson.

Diese Art, von der nur ein $\sigma^{7}$ vorliegt, gehört gleichfalls in die Verwandtschaft von ursina Brsk.

Schwarz, mit Ausnahme des glänzenden Clypeus, matt. Lelzterer ist breit, mit flach gerundeten Vorderecken und schwach ausgebuchtetem Vorderrande. Er ist mässig dicht mit bräunliche Borsten tragenden Punkten bedeckt. Die undeutliche Naht ist bogenförmig. Der Scheitel ist dünn mit feinen anliegenden grauen Haaren bekleidet un trägt abstehende schwärzliche Borstenhaare. Die Fühler sind zehngliederig, der Fühlerschaft ist gelb, der beim $\sigma^{7}$ viergliedrige Fächer schwärzlich. Des Halsschild ist hinten doppelt so breit als lang, am Vorderrande schmäler, die Vorderecken sind nicht vorgezogen, die Bekleidung mit grauen Ü̈rchen ist mässig dicht, die abstehenden schwarzen Haare sind lang und borstenförmig. Die Flügeldecken sind schmal gestreift, die Zwischenräume schwach gewölbt. Sie sind verhältnismässig dünn mit anliegenden schmutziggrauen Härchen bekleidet und tragen namentlich nach hinten zu sehr lang werdende starke schwarze Borsten. Pygidium, Unterseite und Beine sind anliegend greis behaart. Die mit sehr starken und langen Borsten bekleideten Hinterschienen sind ebenso wie bei den verwandten Arten schlanker als bei den übrigen Arten der Gattung Neoserica.

9. Neoserica tonkinensis n. sp. - Opaca, supra fusca, subtus brunnea. Clypeo brunneo, nitido, rugoso, antice attenuato, angulis anticis rotundatis, marginibus elevatis, margine antico haud excïso; fronte nigra, opaca; antennis rufis, 10-articulatis, clava maris 4-, feminae 3- flabellata; prothorace transverso, postice longitudine fere duplo latiore, antice attenuato, haud dense punctato; elytris irregulariter punctatis, striatis, interstitiis subconvexis; pygidio convexo, triangulari, apice rotundato ; pectoris medio abdominisque segmentis fulvo-setosis; femoribus tibiisque posticis modice ampliatis. - Long. 11 mill. - Montes Mauson.

Oben braunschwarz, matt, schwach opalisierend, unten hellbraun. Der Clypeus ist braun, glänzend, nach vorn verjüngt, runzelig punktiert, der Vorderrand fast gerade, die Vorderecken abgerundet. Die gelben Fühler sind zehngliederig, der Fühlerfächer ist beim ${ }^{\lambda}$ 
vier-, beim $q$ dreigliederig. Das Halsschild ist hinten fast doppelt so breit als lang, nach vorn flach bogenförmig verjüngt, die Vorderecken sind etwas vorgezogen, die Oberseite ist fein, mässig dicht punktiert. Die Flügeldecken sind unregelmässig mit augenförmigen Punkten bedeckt, gestreift, die Zwischenräume schwach gewölbt. Das Pygidium ist dreieckig, mit abgerundeter Spitze und schwacher Punktierung. Die Unterseite zeigt ziemlich dicht stehende Augenpunkte, die Nitte der Brust ist zerstreut gelbbraun beborstet und jedes Bauchsegment trägt eine ebensolche Borstenreihe. Die Hinterschenkel und Hinterschienen sind mässig verdickt, letztere aussen mit zwei Borstengruppen.

Die Art hat sehr grosse Aehnlichkeit mit N. soekaranda BRsk. von Sumatra in Grösse, Färbung und Struktur. Die Hinterschenkel und Hinterschienen sind jedoch bei weitem nicht so breit wie bei dieser Art. Die Beschreibung, welche Brenske von N. soekaranda giebt, stimmt nicht genau. Das Kopfschild ist nach vorn verjüngt, glänzend und sehr grob punktiert. Die Z vischenräume auf den Flügeldecken sind nicht fast punktfrei, sondern ebenso wie bei tonkinensis unregelmässig punktiert. Bei der mir unbekannten, aber jedenfalls der tonkinensis ähnlichen N. clypeata FAIRM. von LangSong sind die Zwischenräume der Flügeldecken flach.

10. Neoserica abnormis n. sp. - Oblonga, nigro-brunnea, opaca, paulo opalescens. Clypeo transverso, antice attenuato, marginibus elevatis, margine antico paulo exciso, angulis anticis rotundatis, nitido, fortiter, sparsim punctato, punctis setas fulvas ferentibus, fronte velutina, sparsim setosa; antennis 10 - articulatis, clava maris 6- feminae 4- flabellata; prothorace tranverso, antice attenuato, subtiliter punctato, punctis minutissime setosis; scutello magno, punctato; elytris costatis, irregulariter punctatis, punctis brevissime setosis, costis setis sparsis majoribus ornatis; pygidio paulo convexo, disperse punctato et setoso. Corpore infra punctato, pectoris medio et abdominis segmentis fulvo-setosis; femoribus tibiisque posticis mediocriter dilatatis. - Long. 17 mill. - Montes Mauson.

Eine grosse, längliche Art, die ich, um die Aufstellung einer neuen Gattung zu vermeiden, der Gattung Neoserica zugeteilt habe, obgleich sie von den übrigen Arten dieser Gattung durch den vierblätterigen Fühlerfächer des $q$ abweicht. Sie ist schwarzbraun, einige Exemplare heller, matt, die Unterseite seidenglänzend. Der breite Clypeus ist nach vorn verschmälert, die Ränder sind etwas aufgebogen, der Vorderrand flach ausgebuchtet, die Vorderecken abgerun let. Er ist glänzend braun, zerstreut aber grob punktiert, die Punkte sind mit gelben Borstenhaaren besetzt. Der Scheitel ist 
dunkel, matt, zerstreut borstig behaart. Die braunen Fühler sind zehngliederig. Beim ơ besteht derFühlerstiel aus vier Gliedern und zwar ist das erste Fühlerglied lang, am Ende kugelförmig verdickt und beborstet, das zweite ist knopfförmig, das dritte cylindrisch, das vierte nur halb so lang wie das dritte. Die sechs übrigen Glieder bilden den Fächer. Beim $q$ sind die vier ersten Glieder des Fühlerstieles ebenso gebildet wie beim đ. Das fünfte Glied ist bedeulend kürzer als das vierte und das sechste ist breit, lamellenförmig. Das breite Halsschild ist nach vorn bogenförmig verjüngt; die spitzen Vorderecken sind vorgezogen. Es ist mässig dicht mit sehr kleine Borsten tragenden Punkten bedeckt. Das Schildchen ist gross, dreieckig, die Mitte glatt, die Seiten mit kleinen Borstenpunkten versehen. Die Flügeldecken sind mit unregelmässigen feinen Punkten bedeckt, die äusserst kleine gelbliche Borsten tragen. Die Rippen sind fast glatt und mit in grösseren Abständen stehenden längeren Börstchen geziert. Das etwas gewölbte Pygidium trägt zerstreute beborstete Punkte. Die ganze Unterseite ist mit winzige Borsten tragenden Punkten bedeckt, die Mitte der Brust zeigt einige längere gelbliche Borsten und auf jedem Bauchsegment befindet zich eine Borstenreihe. Hinterschenkel und Hinterschienen sind wenig verbreitert, letztere flach und an den Rändern kräftig beborstet, der Enddorn ist nicht viel kürzer als das erste Tarsenglied. Die Art wurde in Anzahl gesammelt.

11. Pachyserica striatipennis n. sp. - Oblonga, viridifusca, opaca, parce cinereo squamosa. Capite sparsim cinereosquamulato et -piloso, clypeo antice paulo ampliato, angulis anticis rotundatis, marginibus paulo leflexis, margine antico haud exciso; subnitido, punctis raris piligeris; antennis rufis, 10-articulatis; prothorace postice longitudine fere duplo latiore, antice attenuato, lateribus postice sinuatis, disco sparsim versus margines laterales paulo densius cinereo-squamulato; elytris striatis, interstitiis convexis, parce cinereo-variegatis; pygidio corporeque infra sparsim cinereo-squamulatis. - Long. 10 mill. - Montes Mauson.

Die Art, von der nur ein Exemplar vorliegt, hat Aehnlichkeit mit $P$. albosquamosa Brisk. Sie ist etwas grösser, der Clypeus ist grün (bei albosquamosa rotbraun) und der Hinterrand des Halsschildes ist bei weitem weniger nach dem Schildchen zu zurückgezogen. $P$. marmorata BLCH. und rubrobasalis BRsk. sind von striatipennis leicht durch den runzeligen Clypeus zu unterscheiden.

Der Clypeus ist bedeutend breiter als lang, nach vorn schwach erweitert, die Vorderecken sind breit abgerundet, die Ränder etwas aufgebogen, der Vorderrand ist nicht ausgeschnitten. Er ist mit weitläufig stehenden, Borsten tragenden Punkten besetzt. Die Stirn 
ist mit zerstreuten weisslichen Schüppchen bekleidet und dünn abstehend behaart. Die gelbbraunen Fühler sind zehngliederig. Das Halsschild ist hinten sehr breit, nach vorn stark verjüngt. Der Hinterrand ist nach dem Schildchen zu nur schwach zurückgezogen, die Seitenränder sind vor den Hinterecken stark ausgeschweift, die Vorderecken nicht vorgezogen. Die Oberfläche ist dünn, nach den Seitenrändern zu etwas dichter gelblichweiss beschupps. Das Schildchen ist gross, neben den Seitenrändern eingedrückt. Die Flügeldecken sind flach gerippt und mit einigen bindenartigen Zeichnungen aus weisslichen Schüppchen versehen. Am Ende befinden sich neben der Naht einige abstehende kräftige Borsten. Pygidium und Unterseite sind dünn grau beschuppt. Hinterschenkel und Hinterschienen sind schmal, letztere längsgerieft. Die Vorderschienen sind kurz zweizähnig.

12. Gastroserica marginalis Brsk., Mémoires de la Soc. Ent. de Belgique, 1894, p. 51. - Montes Mauson. - Die vorliegenden drei Exemplare sind etwas grösser als die typischen von China. Die Art ist in der Fürbung veränderlich.

Ausser diesen angeführten Serica-Arten liegen noch einige Arten in einzelnen Exemplaren vor, deren Bestimmung mir bisher nicht möglich war.

13. Apogonia cribricollis Bru., IIandb., IV. 2, p. 256, Montes Mauson.

14. Apogonia apicalis n. sp. - Nigro-aenea, nitida, elytrorum margine postico opaco, antennis tarsisque fuscis. Clypeo brevi, integro, subrugoso-punctato, fronte haud dense fortiter punctulata; prothorace transverso, convexo, disco mediocriter lateraliter densius fere ruguloso-punctato; scutello laevi; elytris sat grosse punctatis, lateribus fortiter striatis, supra bigeminatostriatis; pygidio convexo, medio longitudinaliter subcarinato, grosse punctato, griseo-piloso. Subtus medio disperse, lateribus densius punctata; tibiis anticis tridentatis, - Long. 7 mill. Montes Mauson.

Durch Grösse, Gestalt und Form des Clypeus ist die Art der A. laevicollis LsBG. sehr ähnlich. Sie unterscheidet sich jedoch durch kräftigere Punktierung namentlich des Halsschildes und durch den inatten Hinterrand der Flügeldecken. Der Kopf ist breit, die Stirn mässig dicht aber ziemlich grob punktiert, der kurze Clypeus fast bogenförmig, vorn nicht ausgebuchtet. Das gewölbte Halsschild ist grob nadelrissig, an den Seiten dichter, punktiert. Die Flügeldecken sind kurz, kräftig punktiert, neben den Seitenrändern tief punktiert-gestreift, die Naht und zwei von 
Punktreihen eingefasste Linien des Discus sind glatt. Das Pygidium ist grob punktiert, in der Mitte schwach gekielt, mit kurzen abstehenden grauen Härchen besetzt. Die Unterseite ist grob, in der Mitte zerstreut, an den Seitenrändern dichter punktiert, die Punkte tragen kleine weisse Borsten. Die Vorderschienen sind dreizähnig.

15. Apogonia spec? - Montes Mauson. - Es liegen drei Exemplare einer braunen, metallisch schimmernden, mit anliegenden grauen Borstenhaaren bekleideten Art vor. Es ist mir nicht möglich, festzustellen, ob diese Art mit A. cinerascens FAIRu. identisch oder neu ist.

16. Lepidiota bimaculata SAund, Trans. Ent. Soc., II, 1839, p. 76 , t. 16 , f. 2 , - Than-Moi.

17. Lepidiota amœna FeLsche, Deutsche ent. Zeitschr., 1901, p. 360. - Montes Mauson.

18. Lepidiota Brenskei n. sp. - L. nance SHARP affinis. Nigra, subnitida, antennis pedibusque rufis; supra fortiter punctata, brevissime fulvosetosa, subtus pectore fulvo-piloso, abdomine dense fulvo-squamoso. Capite rugoso-punctato, clypeo lateribus paralielis, angulis anticis rotundatis, margine antico haud exciso; prothorace antice attenuato, lateribus post medium fere parallelis, fortiter punctato, breviter fulvo-setoso; elytris quadricostatis, rugoso-punctatis, punctis brevissime fulvo setosis. setis ante apicem lorigioribus; pygidio fulvo squamuloso-setoso, apice conico. Tibiis anticis tridentatis. - Long. 18 mill. - Montes Mauson.

Die Art ist der L. nana SHARP sehr ähnlich, etwas grösser, die Borsten der Oberseite sind viel kürzer und nicht grau sondern schwefelgelb. Der Kopf zeigt eine gleichmässig dichte und grobe Punktierung, die Seitenränder des Clypeus sind parallel, die Vorderecken breit abgerundet, der Vorderrand ist schwach aufgebogen, nicht ausgerandet. Die Seilenränder des Halsschildes sind hinten fast parallel, von der Mitte an verschmälert sich das Halsschild stark noch vorn, der hierdurch gebildete Winkel ist abgerundet. Die spitzwinkligen Vorderecken sind schwach vorgezogen, die Hinterecken sind stumpfwinklig. Die Oberfläche ist grob punktiert, in der Mitte kurz und spürlich nach den Seitenrändern zu länger und dichler gelb beborstet. Das fast halbkreisförmige Schildchen ist, ebenso wie die Flügeldecken, runzelig punktiert und äusserst kurz gelb beborstet. Nur vor dem Hinterrande tragen die Flügeldecken eine Querbinde von längeren schuppenartigen Borsten. Das Pygidium ist mit kleinen ockergelben borstenartigen Schuppen bekleidet, seine Spitze ragt etwas konisch hervor, ähnlich wie bei 
nana. Das Abdomen ist sehr dicht mit länglichen ockergelben Schuppen bedreckt, die Brust lang und zottig mit gelben Haaren bekleidet. Die rotbraunen Beine tragen gelbe Borstenhaare, die Vorderschienen sind dreizähnig.

19. Lepidiota tonkinensis $n$. sp. - I. nanae SHARP affinis. Brunnea, subnitida, antennis pedibusque rufis; supra fortiter, fere ruguloso-punctata, mediocriter fulvo-squamulala, subtus pectore fulvo-villoso, abdomine dense fulvo-squamoso. Capite crebre et fortiter punctato, parce fulvo-squamoso, clypeo lateribus rotundatis, margine antico haud exciso; prothorace antrorsum attenuato, lateribus post medium fere parallelis, ante medium paulo sinuatis, disco sparsim, juxta margines laterales longius et densius, squamoso; elytris quadricostatis, rugoso punctatis, sparsim, ante apicem densius, fulvo-squamulatis ; pygidio dense fulvo-squamoso, apice haud conico. Tibiis anticis tridendatis. - Long. 20 mill. Montes Mauson.

Die Art ist der am gleichen Fundorte gesammelten L. Brenstiei sehr ähnlich, doch abgesehen von der braunen Färbung von dieser Art und auch von nana sofort dadurch zu unterscheiden, dass die Spitze des Pygidiums nicht konisch hervorragt und auch das ganze Pygidium viel flacher ist als bei diesen beiden Arten. Die Gestalt und die Struktur der Oberseite sind fast genau dieselben wie bei Brenskei, nur sind die gelben Schuppen etwas länger und treten dadurch dentlicher hervor. Dae Pygidium zeigt lange anliegende borstenartige Schuppen, welche die Grundfarte völlig verdecken, ähnlich wie bei nana. Behaarung und Beschuppung der Unterseite stimmen mit Brenskei überein.

20. Lepidiota rudepunctata n. sp. - Nigra vel brunnea, subnitida, antennis pedibusque rufis. Supra rugoso-punctata, subtiliter squamulata, subtus pectoris lateribus villosis, abdomine dense fulvo-squamoso. Capite rugoso-punctato, clypeo non emarginato; prothorace ante medium attenuato, rudepunctato, disco sparsim lateraliter densius fulvo-squamoso; elytris rugosopunctatis, quadricostatis, interstitiis subcostatis, punctis brevissime setosis; pygidio dense sulphurea- an cinereo-squamoso; tibiis anticis tridentatis. - Long. : 15 mill. - Montes Mauson.

Diese Art ist mit den vorhergehend beschriebenen Arten verwandt, aber bedeutend kleiner, die Flügeldecken zeigen ausser den vier starken Rippen noch in den Zwischenräumen eine schwach hervortretende Rippe, wodarch die Struktur runzeliger erscheint. Der CIypeus hat parallele Seitenränder, geraden, schwach aufgebogenen Vorderrand, abgerundete Vorderecken. Das Halsschild ist in der Mitte am breitesten, nach hinten kaum, nach vorn ziemlich 
stark verjüngt, der dadurch entstehende Winkel ist abgerundet. Die groben Punkte tragen kleine gelbe Schüppchen, welche neben dem Seitenrande grösser sind und dichter stehen. Die Schüppchen der Flügeldecken sind noch kleiner als die des Halsschildes und nur vor dem Hinterrande befindet sich eine Querbinde von längeren Schuppen. Das flache Pygidium sowohl wie das Abdomen sind äusserst dicht gelb beschuppt, die Seiten der Brust sind behaart. Die Mitte der Brust, die Schenkel und Schienen sind zerstreut beschuppt, die Vorderschienen sind dreizühnig.

21. Asactopholis bituberculata n. sp. - Fusca, supra undique flavo-squamoso-setosa, subtus abdomine haud dense cinereo-squamulato, pectore flavo-piloso. Capite grosse punctato, clypeo margine antico leviter exciso; prothorace lateribus medio parum ampliatis, antice attenuato, dense punctato; elytris tricostatis, aciculato punctatis; pygidio utrinque post medium juxta marginem lateralem tuberculato. Tibiis anticis tridentatis, dente primo minuto. - Long. : 21 mill. - Montes Mauson, Chiem-Hoa.

Hellbraun, die ganze Oberseite licht punktiert, gleichmässig mit schuppenartigen Borsten bekleidet, zwar ziemlich dicht, doch so, dass die Grundfirbung hindurchsieht. Der Vorderrand des Clypeus ist etwas auf - ebogen, schwach ausgeschnitten, die Vorderecken sind stark abgerundet. Die Seitenränder des Halsschildes sind in der Mitte bogenförmig erweitert, die Vorderecken fast rechtwinklig, die IJinterecken stump.winklig, der Hinterrand ist nach dem halbkreisförmigen Schildchen zu bogenförmig zurückgezogen. Die Flügeldecken zeigen zwei flache Rippen auf dem Discus und eine neben dem Seitenrande. Das Pygidium trägt hinter der Mitte jederseits neben dem Seitenrande einen stumpfen Höcker. Das Abdomen ist mit aschgrauen Borstenhaaren bekleidet, welche neben den Seitenrändern dichter stehen, die Brust ist mit langen gelben Haaren bedeckt. Schenkel und Schienen sind gelblich beborstet, die Vorderschienen sind dreizähnig, doch ist der oberste Zahn sehr klein. Die Tarsen sind schlank, das zweite Glied der Hintertarsen ist wie bei den übrigen Arten der Gattung Asactopholis um die Hälfte länger als das erste Cilied. Die Krallen zeigen ausser dem kräftigen Mittelzahn noch ein kleines Basalzähnchen.

22. Gyphochilus apicalis Waterh., Ent. Monthl. Mag., 1867, p. 144. - Montes Mauson.

23. Gyphochilus costulatus BAtes, Ent., XXIV, Suppl., p. 76. - Montes Mauson. - Die Beschreibung, welche BAtes von der Art giebt, passt auf die vorliegenden Exemplare. 
24. Cyphochilus ochraceosquamosus n. sp. - Niger, ntennis pedibusque rufis, supra dense ochraceo-squamosus. Ciapitzsquamoso-setoso, clypeo rotundato; antennis 10-articulatis, clava 3 - articulata, maris elongata; prothorace longitudine duplo latiore, marginibus lateralibus medio incrassatis, antice attenuatis, dense ochraceo-squamoso, squamis setiformibus; elytris 4-costatis, squamulis lanceolatis tectis; pygidio dense punctato, fulvo-squamososetoso. Subtus abdomine setis cinereis squamiformibus medio haud dense, lateraliter densius, tecto, pectore cinereo-piloso, mesosterno inermi; pedibus albo-setosis, tibiis anticis tridentatis. - - Long. : 25 mill. - Montes Mauson.

Etwas kleiner und schmäler als C. costulatus BAtes, die Flügeldecken gleichfalls mit schwach erhabener Naht und vier Rippen, die Seitenränder neben der äussersten Rippe steil abfallend. Auch hat das Mesosternum ebenso wie bei costulatus keinen Fortsatz. Dagegen ist die ganze Oberseite ockergelb beschuppt, die Schuppen sind anders geformt, die am Seitenrande der Flügeldecken sind nicht heller als die übrigen. Die Schuppen des Kopfes und des Halsschildes sind borstenförmig, die der Flügeldecken klein, lanzettförmig, sehr spitz. Das Pygidium ist mit gelben schuppenartigen Borsten bedeckt, doch so, dass die Grundfarhe durchscheint. Die gleichfalls schuppenartigen Borsten des Abdomens, sowie die Haare der Brust sind aschgrau. Die rotbraunen Schenkel und Schienen sind nicht beschuppt, sondern tragen haarförmige weissliche Bosten. Die Vorderschienen sind dreizähnig. Es liegen von dieser Art nur männlich Exemplare vor, bei denen der Fühlerfächer bedeutend länger als der Schaft ist.

25. Gyphochilus podicalis n. sp. - Niger, supra dense fulvo-squamosus, antennis pedibusque rufis. Clypeo rotundato, marginibus paulo elevatis, margine antico haud exciso, antennarum clava maris elongata; prothorace longitudine duplo latiore, lateribus incrassatis, dense fulvo-squamoso; elytris sutura sat elevata, utrinque costis 4 parum elevatis; pygidio fulvis squamis tecto, apice longe acuminato. Subtus abdomine medio-sparsim lateraliter densius fulvo-squamoso, squamis minutis, pectore longe cinereopiloso. Tibiis anticis tridentatis. - Long. 24 mill. - Montes Mauson.

Diese Art, von der gleichfalls nur männliche Exemplare vorliegen, ist der vorhergehenden in Gestalt und Färbung ähnlich. Sie ist dadurch ausgezeichnet, dass das Pygidium am Ende in eine Spitze ausgezogen ist. Es ist nicht ausgeschlossen, dass diese Spitze beim $q$ fehlt. Die Schuppen der Oberseite sind schwefelgelb, die des Kopfes und Halsschildes borstenförmig, die der Flügeldecken 
lanzettformig. Die Flügeldecken zeigen ausser der schwach erhabenen Naht vier weniger dicht beschuppte Rippen und sind die Schuppen des senkrecht abfallenden Seitenrandes heller gefärbt und dichter gestellt. Das Pygidium ist mit kurzen gelben Schuppen bekleidet, in der Mitte flach gekielt und dieser flache Kiel am Ende in eine rötliche Spitze ausgezogen. Das Abdomen ist nicht sehr dicht mit kleinen graugelben Schuppen bekleidet, die Brust mit langer grauer Behaarung versehen. Die Schenkel der roten Beine tragen zerstreute weissliche borstenartige Schuppen. Ein Mesosternalfortsatz ist nicht vorhanden. Die männlichen Fühler sind schiank, der dreigliedrige Fächer ist lang und schmal.

26. Holotrichia sinensis Hope, Trans. ent. Soc., IV, 1845, p. 8. - Montes Mauson.

27. Holotrichia pilosella n. sp. - Nitida, brunnea, capite thoraceque obscurioribus, flavo-pilosa. Capite dense punctato, fronte fortiter carinata, clypeo brevi, antice medio profunde exciso; antennis brunneis, 9-articulatis; prothorace parum transverso, densissime punctato, lateribus crenulatis; elytris grosse, sat dense punctatis, haud costatis; pygidio ruguloso-punctato; abdomine umbilicato-punctato; tibiis anticis tridentatis, articulo primo tarsorum posticorum haud abbreviato. - Long. 20 mill. - Montes Mauson.

Mit II. sinensis Hope verwandt, durch die gelbliche Behaarung leicht von ihr unterschieden.

Braun, Kopf und Halsschild schwärzlich. Der Kopf ist dicht punktiert, abstehend behaart, auf dem Scheitel mit scharfem Kiel. Der Clypeus ist kurz, vorn in der Nitte tief dreieckig ausgeschnitten. Die braunen Fühler sind neungliedrig. Das Halsschild ist etwas breiter als lang, mit schmalem wulstigem Vorderrande und gekerbten Seitenrändern. Die Oberfläche ist äusserst dicht punktiert, kurz abstehend gelblich behaart. Das Schildchen zeigt gleichfalls eine ziemlich dichte feine Punktierung. Die Flügeldecken lassen ausser der schwach erhabenen Naht keine Rippen erkennen. Sie sind gröber und etwas weitläufiger als das Halsschild punktiert, jeder Punkt ist mit einem abstehenden gelblichen Haare versehen. Das Pygidium ist runzelig punktiert, zerstreut behaart. Die Brust trägt eine lange goldgelbe Behaarung, die ziemlich dicht stehenden nadelrissigen Punkte des Abdomens sind mit kürzeren Haaren versehen. Die Schenkel zeigen gleichfalls eine ziemlich dichte Punktierung und gelbe Behaarung. Die Vorderschienen sind kräftig dreizähnig, das erste Glied der Hintertarsen ist annährend so lang als das zweite, der Krallenzahn steht in der Mitte und ist etwas nach rückwärts gebogen. 
28. - Holotrichia tonkinensis n. sp. - Elongata, pruinosa. Capite lato, rugoso-punctato, clypeo rotundato, antice emarginato; antennis brunneis, 10 articulatis; prothorace fortiter punctato, transverso, lateribus integris; elytris late costatis, haud crebre, grosse punctatis, punctis minutissime setosis; pectore dense et longe flavopiloso, abdomine laxe subtiliter punctato; tibiis anticis tridentatis. - Long. 27-30 mill. - Montes Mauson.

Der Holotrichin silikimensis BRSK. sehr ähnlich, durch stärkere Punktierung der Oberseite, sowie durch die ungekerbten Seitenränder des Halsschildes von dieser Art unterschieden. Hierduch unterscheidet sie sich auch von der sehr ähnlichen subiridea FAIR.I. von China.

Der Kopf ist grob, fast runzelig punktiert, der Clypeus vorn ziemlich tief ausgebuchtet. Das Halsschild ist schmal, vorn breit gerandet, die Seitenränder ganz ungekerbt, die Oberfläche ziemlich dicht und grob punktiert mit undeutlicher Mittellinie. Die etwas helleren Flügeldecken sind grob aber nicht dicht punktiert, die Punkte mit winzigen Börstchen besetzt; die Naht und einige Rippen sind schwach erhaben. Das Pygidium ist schwach, etwas runzelig punktiert. Die Brust ist mit langen gelben Haaren dicht besetzt. Das Abdomen ist nur zerstreut und fein punktiert, die Punkte sind mit kleinen Borstenhaaren versehen. Die Vorderschienen sind kräftig dreizähnig. Das erste Glied der Hintertarsen ist etwaś kürzer als das zweite. Der kräftige Zahn der Krallen steht rechtwinklig in der Mitte, die Basis ist verdickt. Die Fühler sind zehngliedrig, das vierte und fünfte Glied sind in der Mitte schwach verbreitert, das sechste und siebente etwas lamellenformig erweitert.

29. Holotrichia bicolorata n. sp. - Opaca, aurantiaca, capite prothoraceque nigrescentibus. Clypeo rotundato, marginibus elevatis, antice profund eexciso, nitido, ruguloso-punctato; fronte opaca, grosse punctulata; antennis 10 - articulatis, funiculo brunneo, clava fulva; prothorace brevi, lateribus integris, sat crebre punctato; scutello lato, punctato; elytris punctatis, tricostatis; pygidio convexo, mediocriter punctulato; pectore flavo-villoso, abdomine punctato, punctis minutissime setosis; pedibus brunneis, nitidis, tibiis anticis tridentatis; articulo primo tarsorum posticorum elongato. - Long. 16 mill. - Montes Mauson.

Die Art ist der $I$. clypealis Brsk., von Kurseong sehr ähalich, doch sind Kopf und Halsschild dunkler gefürbt, die Seiten!änder des letzteren mehr abwärts gebogen, so dass sie von oben betrachtet weniger nach aussen gewinkelt erscheinen, und vor allem ist 
das erste Glied der IIintertarsen mindestens so lang wie das zweite, während es bei clypealis bedeutend kürzer ist.

Gelbrot, Kopf und Halsschild schwärzlich, matt. Der Clypeus ist gerundet, der Vorderrand in der Mitte tief ausgebuchtet, die Oberfläche glänzend, runzelig punktiert. Die Naht ist gerade, die Stirn ist matt, ziemlich grob punktiert. Der Fühlerstiel ist braun, seine Glieder sind kurz, der ovale Fächer ist gelblich. Das Halsschild ist kurz, vorn schmal gerändet, die Seitenränder sind nur vor den Vorderecken schwach gekerbt. Die Oberfläche ist mässig dicht punktiert. Das Schildchen ist breit, fast halbkreisförmig. Die Flügeldecken haben ausser der schwach erhabenen Naht noch drei Rippen, die erste breit, die beiden anderen schmal. Sie sind grob und unregelmässig punktiert. Das Pygidium ist schwach gewölbt, gleichmässig mit genabelten Punkten bedeckt. Die Brust is lang gelblich behaart, das Abdomen mit kleine Borsten tragenden Punkten mässig dicht besetzt. Die Beine sind braun, glänzend, kräftig punktiert. Die Vorderschienen sind dreizähnig, der Krallenzahn ist klein und steht rechtwinklig in der Mitte.

30. Holotrichia Alcocki Brsk., Ind. Mus. Notes, IV, p. 177.Montes Mauson. - Die beiden vorliegenden Exemplare stimmen mit dem typischen Exemplare annährend überein.

31. Holotrichia mausonia n. sp. -- Fusco-nigra, opaca, opalescens. Capite fortiter punctato, flavo-piloso, clypeo brevi, rotundato, antice sinuato; antennis brunneis, clava flava; thorace transverso, punctato, lateribus antice subcrenulatis; elytris subcostatis, fortiter punctatis; pygidio umbilicato-punctato; subtus pectore flavo-piloso, abdomine punctato, punctis minutissime setosis, setis postice longioribus; tibiis anticis tridentatis, articulo primo tarsorum posticorum haud abbreviato, calcare elongato. Long. 21 mill. - Montes Mauson.

Der H. murosa WATERH., von Japan ähnlich, jedoch ist der Kopf behaart, das Halsschild viel kürzer und breiter, seine Punktierung etwas dichter aber schwächer.

Braunschwarz, matt, mit schwachem Seidenglanz. Der Kopf ist grob punktiert, abstehend gelb behaart, der Vorderrand des Clypeus ist schwach ausgerandet. Der Fühlerfächer ist in beiden Geschlechtern sehr klein. Das Halsschild ist kurz und breit, mässig dicht mit Nabelpunkten bedeckt, vorn gerandet, die Randung mit einer Reihe von lange Borsten tragenden Punkten versehen. Die Seitenränder sind in der vorderen Hälfte schwach gekerbt, die spitzen Vorderecken sind stark vorgezogen, der Hinterrand ist an den Seiten schwach wulstig, seine Mitte ist nach dem Schildchen zu etwas 
erweitert. Das letztere ist mit Ausnahme der Ränder punktiert. Die Flügeldecken sind nach hinten weinig verbreitert, ziemlich dicht und grob punktiert, die Naht und einige Rippen treten etwas hervor und sind zerstreuter punktiert. Das Pygidium ist mit nabelförmigen Punkten besetzt. Die Brust ist lang und dicht gelblich behaart, das Abdomen ist mässig dicht punktiert, die Punkte sind mit winzig kleinen börstchen versehen und nur die beiden letzten Bauchsegmente zeigen längere gelbliche Borstenhaare. Die Vorder schienen sind kräftig dreizähnig, das erste Glied der Hintertarsen ist so lang als das zweite, der Enddorn etwas länger als das erste Glied. Der Krallenzahn steht rechtwinklig in der Mitte,

32. Holotrichia Brenskei n. sp. - Fusco-nigra, subnitida. Capite fortiter punctato, clypeo brevi, antice vix sinuato; antennis brunneis, clava maris parva, ovata; prothorace sat dense punctato, lateribus antice margineque postico crenulatis; elytris subcostatis, fortiter punctatis, punctis brevissime setosis; pygidio nitido, disperse punctato. Subtus pectore villoso, abdomine medio laxe, lateribus paulo densius punctato, punctis setas minutas ferentibus; tibiis anticis tridentatis, tarsis piceis, articulo primo torsorum posticorum secundo paulo longiore. - Long. 18 mill. - Montes Mauson.

Diese Art, von der nur ein männliches Exemplar vorliegt, hat in der Gestalt einige Aehnlichkeit mit $H$. anthracina BRsk., unterscheidet sich aber durch die Struktur und die Länge des ersten Tarsengliedes der Hinterbeine.

Schwarz, die Beine brüunlich. Der Kopf ist müssig stark punktiert, der Clypeus sehr kurz, sein Vorderrand in der Mitte kaum sichtbar ausgebuchtet. Die braunen Fühler haben einen kurzen Fächer. Das Halsschild ist ziemlich dicht und grob punktiert, aber bei weitem nicht so grob wie bei anthracina. Es ist vorn schmal gerandet, die Seitenränder sind nur vor den Vorderecken sehr schwach gekerbt, wogegen der ganze Hinterrand eine feine Crenulierung zeigt. Das Schildchen trägt nur einige kleine zerstreute Punkte. Die Flügeldecken zeigen ausser der schwach erhabenen Naht nur einige undeutliche Rippen. Sie sind grob, aber nicht sehr dicht punktiert, die Punkte tragen ein kaum sichtbares kleines Börstchen. Das glänzende Pygidium zeigt weitläufig stehende Punkte. Die Brust ist gelblich behaart, das Abdomen in der Mitte sehr zerstreut, nach den Seiten zu etwas dichter punktiert, die Punkie mit kleinen Borsten besetzt. Die Vorderschienen sind kräftig dreizähnig, das erste Glied der Hintertarsen ist fast länger wie das zweite, der eine Enddorn überragt etwas das erste Tarsenglied. 
Endzahn und Krallenzahn bilden einen spitzen Winkel und ist der letztere kräftiger.

33. Holotrichia lata Brsk., Berlin. ent. Zeitschr., 1892, p. 163. - Than-Moi, Montes Mauson.

34. Holotrichia Severini Brsk., Mém. Soc. ent. Belgique, II, p. 62. - Montes Mauson. - Ich halte die Exemplare von Tonkin nicht für verschieden von den typischen von Java. Die Exemplare von Tonkin sind durchschnittlich etwas robuster als die von Java. Sie variiren in der Färbung von dunkelbraun bis hellbraun und ist meist das Ha!sschild etwas dunkler. Auch Struktur und Körperform sind bei den einzelnen Exemplaren verschieden.

35. Holotrichia iridescens n. sp. - Nitida, nigra an brunnea, plus minusve violaceo-micans. Capite grosse et dense punctato, clypeo sinuato; antennis brunneis, 10-articulatis, clava in utroque sexu parva; prothorace fortiter aciculato-punctato, linea media indistincta laevi, lateribus crenulatis; scutello, medio excepto, punctato; elytris subcostatis, grosse umbilicato-punctatis; pygidio convexo, sat dense punctato. Pectore flavo-piloso, abdomine umbilicato-punctato, punctis minutissime setosis, segmento penultimo lateraliter ultimoque postice flavo-ciliatis; femoribus fortiler punctatis, tibiis anticis tridentatis, articulo primo tarsorum posticorum haud abbreviato; unguibus dente medio acuto. - Long. : 24 mill. - Montes Mauson, Than-Moi.

Braune Exemplare haben Aehnlichkeit mit $H$. pygidialis Brsk., von Borneo, doch ist die neue Art robuster, die Punktierung des Halsschildes ist viel dichter, das letzte Abdominalsegment hat keinen zapfenartigen Vorsprung.

Schwarz oder kastainenbraun, mehr oder weniger violett schimmernd. Der Kopf ist grob und dicht, der Clypeus meist runzelig punktiert, vorn ausgerandet, die Vorderecken sind stark abgerandet. Die braunen Fühler haben in beiden Geschlechtern einen sehr kleinen Fächer, doch ist der des $\sigma^{\top}$ etwas länger. Das Halsschild ist ziemlich dicht nadelrissig punktiert mit mehr oder wenig deutlicher glatter Mittellinie, vorn schmal gerandet, die Seiten sind schwach gekerbt, die vorgezogenen Vorderecken fast rechtwinklig. Das Schildchen hat eine glatte Mittellinie. Die Flügeldecken sind nach hinten etwas verbreitert, tief nabelförmig punktiert, die Naht und vier sehr flache, schmale Rippen etwas glatter. Das Pygidium ist schwach gewölbt und mässig dicht mit Nabelpunkten bedekt. Die Brust ist mit Ausnahme der Mitte gelblich behaart, das Abdomen zeigt nicht sehr dicht stehende nadelrissige Punkte, die winzige Börstchen tragen. Das vorletzte Bauchseg- 
ment ist vor dem Hinterrande eingeschnürt, das letzte Bauchsegment ist beim $\sigma^{\gamma}$ am Vorderrande sehr deutlich gewulstel. Beim q tritt diese Wulst weniger deutlich hervor, weil das ganze letzte Bauchsegment gewölbt ist. Die Vorderschienen sind kräftig dreizähnig, das erste Glied der Hintertarsen ist nicht kürzer als das zweite, der eine Enddorn bedeutend länger als das erste Tarsenglied. Der Klanenzahn steht rechtwinklig in der Mitte.

36. Holotrichia subsericea n. sp. - Ochracea, subsericea. Clypeo dense punctato, brevi, rotundato, antice vix emarginato; fronte fortiter, sparsim punctata, fulvo-pitosa; antennis rufis, 10 -articulatis; prothorace lato, punctato, lateribus antice subcrenulatis et setosis; elytris indistincte bicostatis, punctatis; pygidio ruguloso-punctato. P'ectore flavo-piloso, abdomine opaco, punctato, segmento penultimo feminæ in medio tuberculato; tibiis anticis tridentatis, articulo primo tarsorum posticorum haud abbreviato, unguibus apice profunde fissis. - Long. 18 mill. - Montes Mauson.

Die Art ist der H. aurosericea Brsk. von Tenasserim sehr ähnlich und steht auch bei ihr der Mittelzahn der Krallen der Spitze nahe und läuft mit dem Spitzenzahn parallel, so dass die Klauen tief gespalten erscheinen. Die Art ist leicht daran zu erkennen, dass das $q$ auf der Mitte des vorletzten Bauchsegments einen blasenartigen, flach längsgefurchten, glänzenden Höcker trägt.

Die Farbe ist braungelb, seidenartig. Der Clypeus ist dicht punktiert, kurz, gerundet, vorn kaum ausgeschnitten. Die Stirn ist zerstreut grob punktiert und mit abstehenden braunen Haaren besetzt. Die Fühler sind zehngliedrig, der dreigliedrige Fächer ist beim ơ so lang wie die sechs vorhergehenden Glieder zusam men, beim $q$ ein weinig kürzer. Das Halsschild ist mässig dicht punktiert, vorn schmal gerandet, die Seitenränder in der vorderen Hälfte nur ganz schwach gekerbt und mit einzelnen Borsten besetzt. Die Flügeldecken zeigen ausser der deutlichen Naht nur noch zwei schwache Rippen und sind gleichmässig dicht punktiert. Die Punktierung des Pygidiums ist runzlig. Die Brust is lang gelb behaart, der aufgeblasene Bauch etwas dunkler gefärbt und malt, der ơ zeigt auf der Mitte des vorletzten Bauchsegments sta!l des Tuberkels einen glänzenden Fleck. Die Vorderschienen sind dreizähnig, das erste Glied der Hintertarsen ist so lang als das zweite, die Enddornen sind kürzer als das erste Farsenglied.

37. Holotrichia planicollis Buru., Handb., IV, 2, p. 352.Montes Mauson, Chiem Hoa.

38. Holotrichia cephalotes Burm., Handb., IV, 2, p. 352.Montes Manson, Chiem Hoa. Die Art steht der H. planicollis Buru. 
sehr nahe, unterscheidet sich aber dadurch, dass der kräftige klauenzahnn icht wie bei planicollis dem Endzahn nahe gerückt ist und mit diesem einen spitzen Winkel bildet, sondern in der Mitte fast rechtwinklig steht.

RertTer hat in seiner Bestimmungstabelle der Melolonthiden der europäischen Fauna auf Grund der 14 Holotrichia Arten, welche er anführt, (ca 150 Arten sind beschrieben!) 3 neue Untergattungen aufgestellt und so auch auf $H$. planicollis Burm (nigra ReDTB.) die Gattung Eretusa wegen der Klauenbildung errichtet. H. cephalotes Buru., welche ReITTER nicht kennt, hat anders gebildete Klauen und könnte danach trotz ihrer nahen Verwandschaft mit planicollis nicht mit dieser in dieselbe Untergattung kommen. Herr ReITTER hätte besser getan, nicht durch die Aufstellung seiner Untergattungen dem Spezialisten die Arbeit zu erschweren. Was hat Microtrichia Standfussi Brsk. von Sumatra, worauf die Gattung Noanda errichtet ist, in einer Bestimmungstabelle der europäischen Melo lonthiden zu suchen?

39. Brahmina chinensis Brsk., Berlin ent. Zeitschr., 1892, p. 101. - Montes Mauson.

40. Brahmina tonkinensis n. sp. - Oblonga, supra fusca, nitida, subtus brunnea, lateribus pruinosis. Capite grosse et dense punctato, clypeo antice emarginato; antennis brunneis, 10-articulatis; prothorace fortiter sat crebre punctato, linea media obsoleta laevi, lateribus subcrenulatis; elytris grosse punctatis, sutura costisque duabus paulo elevatis ; pygidio dense umbilicato-punctato ; corpore infra sparsim punctato, pectoris abdominisque lateribus opacis, pruinosis; tibiis anticis tridentatis, articulo primo tarsorum posticorum abbreviato. - Long. 15 mill. - Montes Mauson.

Der B. thoracica Brsk. von Kurseong ähnlich, etwas grösser, das Halsschild vor dem Hinterrande nicht eingeschnürt.

Die Oberseite ist schwarzbraun, glänzend, die Unterseite hellbraun. Die ganze Oberseite ist kräftig, ziemlich dicht punktiert. Das Kopfschild ist vorn ziemlich tief ausgeschnissen. Die braunen Fühler sind zehngliedrig, der Fächer ist beim $\sigma^{\lambda}$ so lang wie die sechs vorhergehenden Glieder zusammen, beim q etwas kürzer. Das Halsschild zeigt in der Mitte eine vorn und hinten verkürzte glatte Längsschwiele, vorn ist es schmal gerandet, an den Seiten nur undeutlich gekerbt. Die Flügeldecken zeigen ausser der etwas erhabenen Naht zwei schmale Rippen. Die Unterseite ist nicht dicht punktiert, in der Mitte glänzend, an den Seiten pruinös. Die Vorderschienen sind dreizänig, das erste Glied der Hintertarsen ist etwas kürzer als das zweite. Der kräftige Mittelzahn der Klauen ist dem 
schwächeren Endzahn nahe gerückt und läuft mit diesem parallel, so dass die Klauen an der Spitze gespalten erscheinen.

41. Brahmina pumila ShArp? Notes Leyd. Mus, III, p. 225. - Montes Mauson. - Die Exemplare von Tonkin sind etwas grösser als meine Exemplare der pumila von Java und ist auch das Halsschild etwas runzeliger punktiert, doch glaube ich, dass sie derselben Art angehören. Merkwürdig ist es, dass von den drei vorliegenden Exemplaren von Tonkin zwei neungliedrige Fühler haben.

42. Hoplosternus chinensis GuÉr., Voy. Favor., p. 63, t. 232, f. 3. - Montes Mauson.

43. Hoplosternus laevipennis Blanch., Cat. Coll. Enl., 1850, p. 158. - Montes Mauson. - Von dieser Art liegen zwei Pärchen vor. Bei den $\sigma^{\top}$ ist die ganze Oberfläche mit graubraunen, bei den $q$ mit hellgrauen Schuppenhaaren besetzt. Das Halsschild ist in der Mitte dunkler gefärbt.

44. Melolontha mandarina Sharp, Col. Hefte, XIV, p. 87. Montes Mauson. - Auf die vorliegenden Exemplare passt die Beschreibung, welche SiARp von der Art giebt. Die Art ist der serrulata GyLL. sehr ähnlich, unterscheidet sich aber unter anderem durch die in beiden Geschlechtern sehr kurzen Fühlerfächer. 


\section{$2 \mathrm{BHL}$ Biodiversity Heritage Library}

Moser, Josef. 1908. "Verzeichnis der von H. Fruhstorfer in Tonkin gesammelten Melolonthiden." Annales de la Société entomologique de Belgique 52, 325-343. https://doi.org/10.5962/bhl.part.5012.

View This Item Online: https://www.biodiversitylibrary.org/item/47476

DOI: https://doi.org/10.5962/bhl.part.5012

Permalink: https://www.biodiversitylibrary.org/partpdf/5012

\section{Holding Institution}

Smithsonian Libraries

\section{Sponsored by}

Smithsonian

\section{Copyright \& Reuse}

Copyright Status: NOT_IN_COPYRIGHT

This document was created from content at the Biodiversity Heritage Library, the world's largest open access digital library for biodiversity literature and archives. Visit BHL at https://www.biodiversitylibrary.org. 